MATHEMATICS OF COMPUTATION

Volume 76, Number 260, October 2007, Pages 1925-1940

S 0025-5718(07)01992-8

Article electronically published on May 30, 2007

\title{
A MODEL FOR PEAK FORMATION IN THE TWO-PHASE EQUATIONS
}

\author{
BJÖRN SJÖGREEN, KATARINA GUSTAVSSON, AND REYNIR LEVI GUDMUNDSSON
}

\begin{abstract}
We present a hyperbolic-elliptic model problem related to the equations of two-phase fluid flow. The model problem is solved numerically, and properties of its solution are presented. The model equation is well-posed when linearized around a constant state, but there is a strong focusing effect, and very large solutions exist at certain times. We prove that the model problem has a smooth solution for bounded times.
\end{abstract}

\section{INTRODUCTION}

Mathematical modeling and numerical approximation of industrial processes involving two-phase flow has become an important area of research. Much effort is currently devoted to develop physical and mathematical models for these kinds of flows.

Mathematical models of a fluid with dispersed particles can be formulated in different ways, depending, on e.g., the application and the number of particles in the suspension.

In this paper we are concerned with a two-phase flow model that treats the flow on a macroscopic level and phases are considered as interacting continua. The model consists of two coupled sets of conservation equations for mass and momentum for each phase and the final set of equations resembles a Navier-Stokes system. This is sometimes referred to as the Eulerian/Eulerian approach and is discussed, e.g., in [3, 5, 16].

This model of a two-phase flow have many interesting features. For example, it was shown in [6, 18, that the equations in the inviscid limit, are ill-posed when linearized around states belonging to a certain set in phase space. In [18] the viscous formulation is also studied and it is shown that for smooth initial data in this region of phase space, exponential increase of the solution does occur when the viscous term is small. A study of a model problem with similar behavior is considered in 11. Nevertheless, the equations are solved by engineers [17, and used in prediction of flow behavior in machinery relevant to, e.g., process industry. A bounded solution can exist even if it develops non-smoothness. For example, it is known that shock waves can have a regularizing effect by absorbing small oscillations. Another possible mechanism is that instabilities quickly force the solution to leave the region of ill-posedness. In this context, one could also

Received by the editor June 20, 2005 and, in revised form, June 4, 2006.

2000 Mathematics Subject Classification. Primary 76T25, 65M99, 35L60.

(C)2007 American Mathematical Society

Reverts to public domain 28 years from publication 
imagine a situation where the solution oscillates periodically between ill-posed and well-posed states.

A model problem with an ill-posed region of phase space was presented in [18]. The computations in [8] and in [18, showed the appearance of highly oscillatory solutions for the one-dimensional two-phase equations. The frequency of the oscillations grew as a regularizing viscosity parameter was sent to zero. Questions regarding the regularity of the solution and the limit behavior of the solution of the regularized system are still open.

We present here a hyperbolic-elliptic model problem where only a few terms of the one-dimensional two-phase equations are retained. The model problem is wellposed for all states, but nevertheless shows strong growth and formation of smallscale features. Therefore, one should not claim that all interesting phenomena in the two-phase system stems from the ill-posed inviscid limit.

A closely related equation occurring in chemotaxis and chemosensitive movement was analyzed in [10].

In Section 2, we recall some results from [8] for the two-phase equations in one space dimension. In Section 3, we simplify the equations to obtain the model problem. The properties of the solution of the model problem are demonstrated by numerical experiments. Finally, analysis presented in Section 4 shows that the model problem with compact data on an infinite domain has a smooth solution for bounded times.

\section{Formation of PEAKS IN THE TWO-PHASE EQUATIONS}

We consider a two phase flow consisting of a particle phase and a fluid phase. Both phases are assumed incompressible. The following model describes the evolution of the two phases,

$$
\begin{aligned}
\phi_{t}+\nabla \cdot \phi \mathbf{u} & =0 \\
\rho_{p}(\phi \mathbf{u})_{t}+\rho_{p} \nabla \cdot\left(\phi \mathbf{u u} \mathbf{u}^{T}\right) & =-\phi \nabla p-G(\phi) \nabla \phi \\
& -K(\phi)(\mathbf{u}-\mathbf{v}) \\
& +\mathbf{g} \rho_{p} \phi+\nabla \cdot\left(\mu_{p} \phi D_{p}\right), \\
\rho_{c}((1-\phi) \mathbf{v})_{t}+\rho_{c} \nabla \cdot\left((1-\phi) \mathbf{v} \mathbf{v}^{T}\right) & =-(1-\phi) \nabla p \\
& +K(\phi)(\mathbf{u}-\mathbf{v}) \\
& +\mathbf{g} \rho_{c}(1-\phi)+\nabla \cdot\left(\mu_{c}(1-\phi) D_{c}\right), \\
\nabla \cdot(\phi \mathbf{u}+(1-\phi) \mathbf{v}) & =0 .
\end{aligned}
$$

Here $\phi$ denotes the particle volume fraction, $\rho_{p}, \rho_{c}$ are the constant densities of the particle $(p)$ and fluid $(c)$ phases, respectively. $\mathbf{u}, \mathbf{v}$ are the particle and fluid velocities, $D_{p}, D_{c}$ are the symmetric part of the particle and fluid velocity gradients, e.g.,

$$
D_{p}=\frac{1}{2}\left(\nabla \mathbf{u}+(\nabla \mathbf{u})^{T}\right) .
$$

The fluid pressure is denoted by $p$ and $G(\phi)$ is the modulus of elasticity for the particulate phase. $K(\phi)$ is a particle-fluid friction term and $\mathbf{g}$ is gravity, $\mu_{p}$ and $\mu_{c}$ are viscosity coefficients of the particle and fluid phases. There exist many different models for the material functions $G$ and $K$. In the first part of this paper, when 
we are solving (10), we use the simplified models

$$
\begin{array}{r}
G(\phi)=0.02\left(\frac{\phi}{\phi_{m p}-\phi}\right)^{3}, \\
K(\phi)=\frac{17.3 \mu_{c}}{d_{p}^{2}} \cdot \frac{\phi}{\left(\phi_{m p}-\phi\right)^{2.8}},
\end{array}
$$

where the particle diameter $d_{p}=0.0005$, and the maximum packing $\phi_{m p}=0.8$; see 8.

We consider the one-dimensional version of (11) on the domain $-1.5 \leq x<1.5$. In $[18$ it is shown that this problem is locally in time well-posed for smooth data, but with exponential growth in time for data in a certain region in phase space. The boundary conditions are periodic and the initial data is chosen such that $\phi$ is a positive and periodic function

$$
\phi(x, 0)=0.2 \sin \frac{2 \pi x}{3}+0.25 .
$$

The initial velocities are assumed in equilibrium, i.e., such that the zero order terms in the equations are zero. This condition together with the incompressibility constraint give, in one space dimension,

$$
u=\phi(1-\phi)^{2}\left(\rho_{p}-\rho_{c}\right) g / K(\phi) \quad \phi u+(1-\phi) v=0 .
$$

This means that both the initial particle velocity, $u$, and the initial fluid velocity $v$, are determined from the initial data for $\phi$. This set of initial data is in the above mentioned growth region in phase space. To investigate whether a constant state is in the growth region, one linearizes the equations around that state, and takes the Fourier transform. One linear ordinary differential equation is obtained for each Fourier mode. In the growth region, all Fourier modes increase exponentially, but with a rate which is bounded uniformly with respect to the wave number.
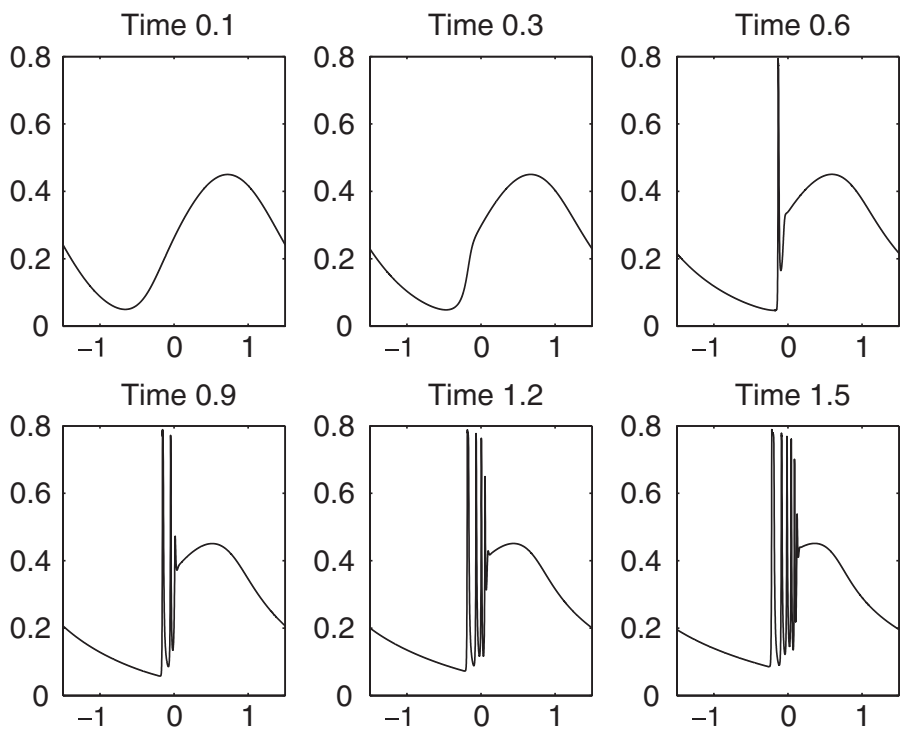

FIgURE 1. Time evolution of $\phi .4000$ grid points. 


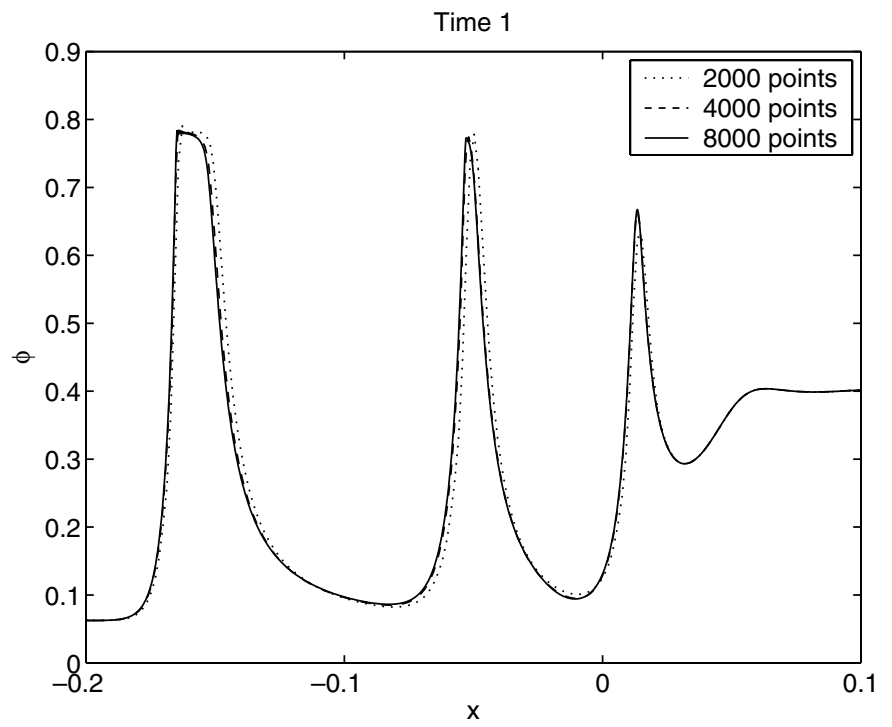

Figure 2. Solution at time 1. Close-up of the oscillations. Grids of 2000,4000 , and 8000 points.

In [8], this problem was solved by a spectral approximation in space and a second order Adams predictor-corrector method in time. Here, we instead use a sixth order accurate centered difference approximation in space, all derivatives $\partial / \partial x$ are replaced by the divided difference operator (6). To make sure non-linear stability holds, a small eight order dissipative term is added to each equation. This term does not affect the order of accuracy. A fourth order Runge-Kutta scheme is used for time integration. The problem parameters are set to the following values $\rho_{p}=200, \rho_{c}=1, \mu_{p}=1, \mu_{c}=1.8 \times 10^{-5}$.

In Figure 1 we present the volume fraction, $\phi$, at different times. The sine wave first breaks and forms a steep gradient. A sharp peak is formed at the gradient. Secondary peaks are formed, which at later times interact, and eventually fill up the entire axis with oscillations. Once oscillations are formed they will increase, due to the high wave number growth of the linearized problem. The oscillations can stabilize due to non-linear effects, or because they reach values that are outside the growth region in phase space. Closer investigation of the oscillations shows that the width of the peaks is never smaller than approximately 20 particle diameters. For a more complete study of this problem, see [7.

Note that this is a viscous problem, but it is not evident that a smallest scale exists. However, the grid convergence study shown in Figure 2 gives an indication that it is possible to resolve all scales, at least up to a bounded time. In Figure 2 we display a close-up of the oscillations in $\phi$ at time 1 . The grids have size 2000, 4000, and 8000 grid points. The solution does not change visibly between the two finest grids; grid convergence seems to have been attained.

We will next present a linearly well-posed model problem which retains some features of the solution of (11). The purpose of this section was to show some properties (1) and to motivate the need for models that give insight into oscillatory phenomena of these equations. 


\section{A MOdEL PROBLEM}

The one-dimensional version of the two-phase equations, as formulated in (1), are often used as a model of gravity dominated sedimentation processes. When the flow is gravity dominated and slow, the transport terms in (1) can be neglected. Also, if the suspension is such that its mixture viscosity is orders of magnitude larger than the viscosity of the fluid, we can ignore the fluid stress tensor, $\mu_{c}(1-\phi) D_{c}$. In this case we assume that the mixture viscosity equals the particle viscosity. Following these assumptions and using the fact that the fluid pressure can be eliminated by direct integration, we obtain

$$
\begin{aligned}
\phi_{t}+(\phi u)_{x} & =0 \\
\left(\mu_{p} \phi u_{x}\right)_{x}-\frac{K}{(1-\phi)^{2}} u & =G(\phi) \phi_{x}-\Delta \rho g \phi
\end{aligned}
$$

to be solved for the particle phase velocity $u$ in the $x$-direction and the volume fraction $\phi$.

If $\mu_{p}=0$, this is a scalar conservation law for the volume fraction, $\phi$. This is the classical, inviscid, 1D consolidation model and has been discussed in many important works; see e.g. [1, 2, 4.

In this study we work with a slightly different model, the viscous model, where $\mu_{p} \neq 0$. This term is small and usually ignored in the classical way of treating this problem. However, from a numerical point of view, the viscous model is more advantageous since a less restrictive condition on the time step (CFL condition) is obtained than with the classical model. This is indicated by a linear stability analysis and exemplified by numerical experiments presented in [9].

During a typical sedimentation process the solid phase will be separated from the fluid phase, and numerical experiments as well as experimental data show a steep gradient in the volume fraction at the clear fluid interface. In the clear fluid region the modulus of elasticity is equal to zero and the equation for the volume fraction in the classical approach is hyperbolic, and mathematically the regions are separated by a shock. However, the viscous model gives a volume fraction profile with a very steep gradient at the interface.

To investigate whether this is a shock or not we will study a model problem obtained by removing the the modulus of elasticity, $G(\phi)$, from (21). Also, we denote $\mu_{p} \phi=\eta$ and let $\eta$ be a constant, set $K /(1-\phi)^{2}=1$ and $\Delta \rho g=1$. This yields the following model problem:

$$
\begin{aligned}
& \phi_{t}+(u \phi)_{x}=0, \quad 0<x<L \quad t>0 \\
& -\eta u_{x x}+u=\phi
\end{aligned}
$$

The model is considered together with periodic boundary conditions and periodic initial data, $\phi(x, t)=\phi_{0}(x)$. The particle phase velocity $u$ depends on $\phi$ through the elliptic equation (4). When the viscosity parameter $\eta$ is zero, this is exactly the inviscid Burgers' equation for $\phi$. It is well known that this equation has a bounded, but discontinuous solution; see [13].

Surprisingly, the viscous term $\eta u_{x x}$ in (4) does not act as a smoothing. Linearization around the constant states $u=u_{0}$ and $\phi=\phi_{0}$ and Fourier transformation gives

$$
\hat{\phi}_{t}+i \xi \frac{\phi_{0}}{1+\eta \xi^{2}} \hat{\phi}=0
$$


where $\xi$ is the frequency. Here we see that the viscosity only introduces a frequency dependent change in the propagation speed. However, the behavior of the solution of the non-linear problem is different from what is indicated by the linear analysis. Writing the $\phi$-equation, (3), as

$$
\phi_{t}+u \phi_{x}=-u_{x} \phi
$$

shows the growth term $u_{x} \phi$, which is zero when linearized. However, for $u_{x}<0$, strong growth in $\phi$ takes place.

We first study equations (3) and (4) by some numerical experiments, which will give insight into the properties of the solution. We approximate the derivative in space in equation (3) by a sixth order accurate difference operator to get the semi-discrete approximation

$$
\frac{d \phi_{j}(t)}{d t}+D_{06}\left(u_{j} \phi_{j}\right)=0
$$

with the operator $D_{06}$ defined as

$$
D_{06} v_{j}=\frac{-v_{j-3}+9 v_{j-2}-45 v_{j-1}+45 v_{j+1}-9 v_{j+2}+v_{j+3}}{60 \Delta x} .
$$

The grid is uniform with grid spacing $\Delta x=x_{j+1}-x_{j}$. Periodic boundary conditions are imposed, so that boundary modifications of the difference operators are not necessary. The velocity $u_{j}$ in (5) is computed by solving equation (4) numerically as

$$
-\eta D_{6} u_{j}+u_{j}=\phi_{j}
$$

which is an invertible linear system of equations under periodic boundary conditions. The sixth order accurate approximation of the second derivative is given by

$$
\begin{aligned}
D_{6} v_{j}= & \left(\frac{1}{90} v_{j-3}-\frac{3}{20} v_{j-2}+\frac{3}{2} v_{j-1}-\frac{49}{18} v_{j}\right. \\
& \left.+\frac{3}{2} v_{j+1}-\frac{3}{20} v_{j+2}+\frac{1}{90} v_{j+3}\right) / \Delta x^{2} .
\end{aligned}
$$

Since (5) is of the form $\phi^{\prime}(t)=f(\phi)$, we solve this ODE in time by the standard fourth order accurate Runge-Kutta method.

For the numerical experiments, we use the initial data

$$
\phi(x, 0)=0.9 \sin \pi x+1
$$

and we set $\eta=0.01$. The computational domain is $0 \leq x<2$, with the periodic boundary conditions, $\phi(0, t)=\phi(2, t)$. Motivated by the physical interpretation, we will only consider non-negative $\phi$. It is not hard to see, by rewriting the equation as an ODE along a characteristic,

$$
\frac{D \phi}{D t}=-u_{x} \phi
$$

(where $D / D t=\partial / \partial t+u \partial / \partial x$ ), that if the initial data is non-negative, then the solution will stay non-negative for all times.

The time evolution of a typical solution obtained for $\eta=0.01$ is shown in Figure 3. We see how the solution focuses to a spike-like feature at time 0.8 , which is reduced but re-emerges at a later time. The velocity corresponding to the solution in Figure 3 is shown in Figure 4. The computations show a bounded velocity, but with a possible jump in the derivative $u_{x}$. 

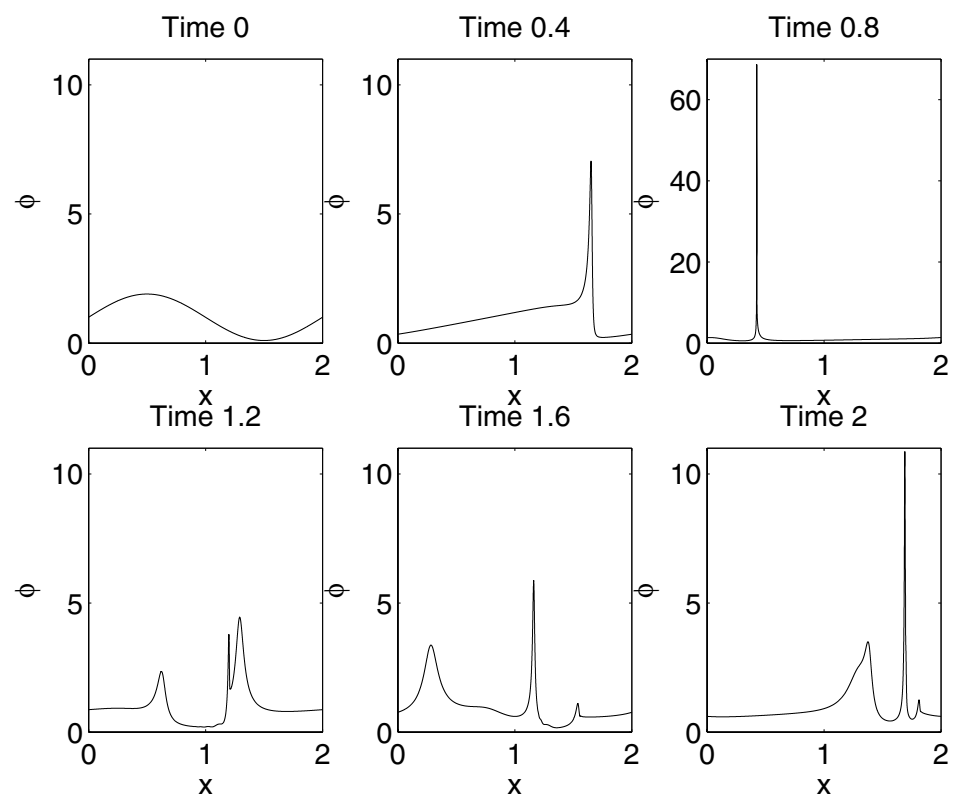

Figure 3. Time evolution of $\phi$, test problem.
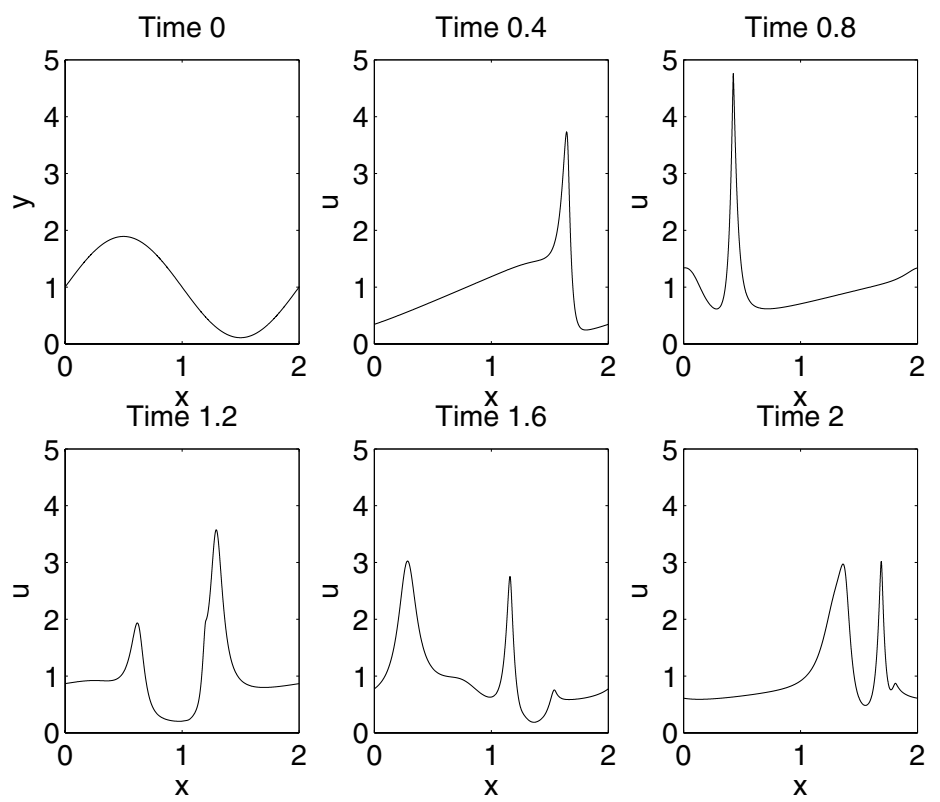

Figure 4. Time evolution of $u$, test problem. 

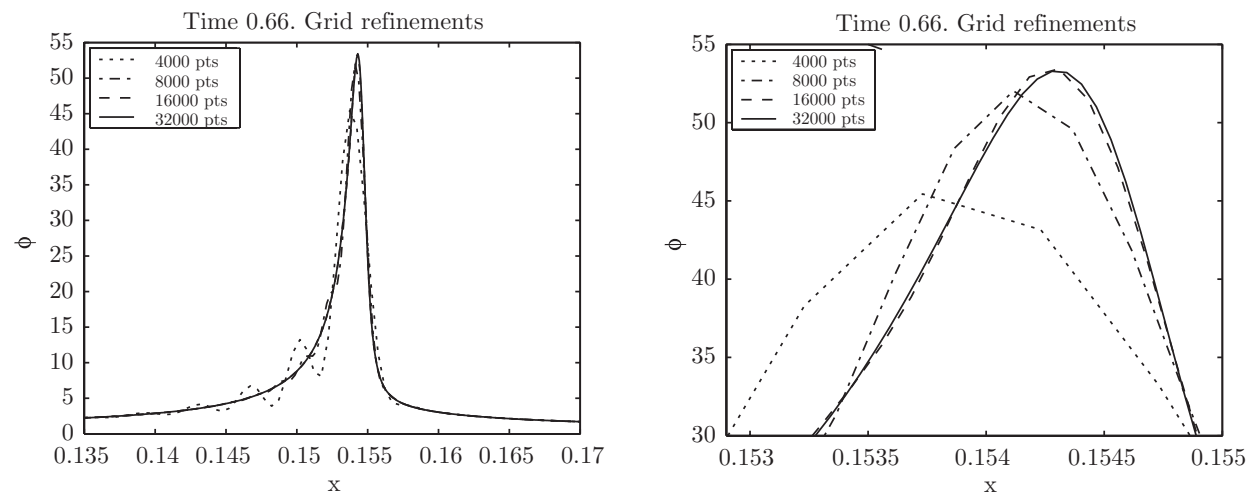

Figure 5. Grid refinement study of $\phi$ at time 0.66. The right figure shows the same as the left, but with a smaller scale.

The question is whether the solution is singular at the spike. Integrating the $\phi$-equation over one period shows that

$$
\int \phi(x, t) d x=\int \phi(x, 0) d x
$$

which, since $\phi \geq 0$, means that $\phi$ could possibly blow up at a point $x_{0}$, but with a rate less than $\approx 1 /\left(x-x_{0}\right)$.

Figure 5. shows a grid refinement study of the solution at $t=0.66$, which is very close to the time at which the solution attains its maximum value. The solution is shown only in a region near the spike. When refining up to 16000 grid points, the solution keeps increasing, but when refining from 16000 to 32000 grid

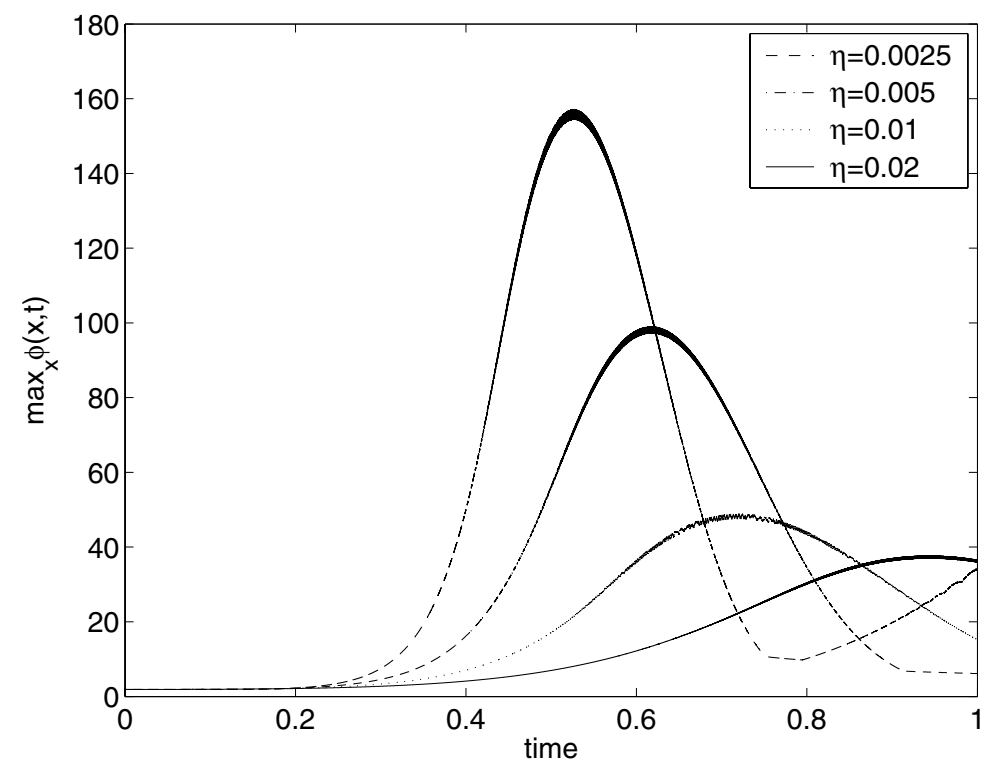

FiguRE $6 . \max _{x} \phi$ vs. time for $\eta=0.02$ (solid), $\eta=0.01$ (dot), $\eta=0.005$ (dash-dot), and $\eta=0.0025$ (dash). 


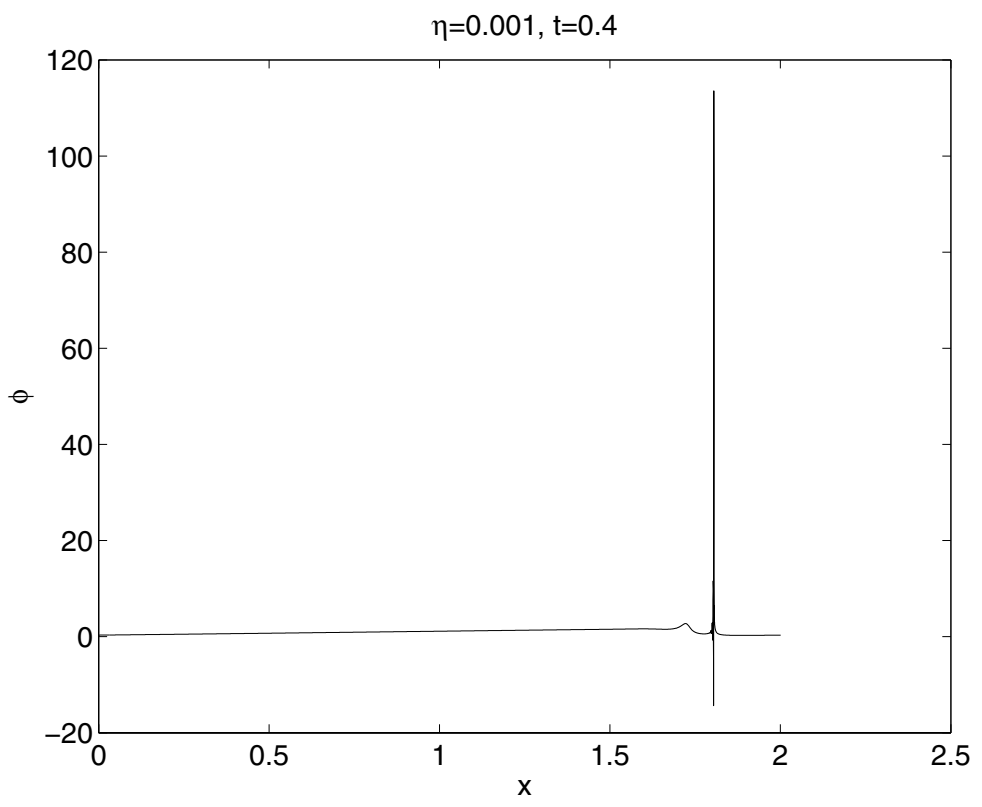

Figure $7 . \quad \phi$ at time 0.4 for $\eta=0.001$.

points, the maximum value of the solution is unchanged and the solution curves are almost indistinguishable. We conjecture that the solution does converge to a smooth function as the grid is refined and that the spike-like phenomenon is not a singularity. Another illustration of the increase in $\phi$ is shown in Figure 6, where $\max _{x} \phi(x, t)$ is plotted versus time for the same computations as shown in Figure 3, In Figure 6, we also show the growth for computations with $\eta=0.0025, \eta=0.005$, and $\eta=0.02$, but which are otherwise identical to the computation with $\eta=0.01$.

The solution for $\eta=0.001$ at the time 0.4 is displayed in Figure 7. The focusing is very strong, and the solution is not resolved on the grid used. The peak value is approximately 120 , but from the experience with $\eta=0.01$ we expect the grid converged peak value to be even higher.

We conclude that the solution becomes larger when $\eta$ becomes smaller. Convergence of $\phi$ to the solution of Burgers' equation in the limit $\eta \rightarrow 0$ is questionable. The model problem given by equations (3) and (4) is a suitable test problem for grid adaptive methods.

3.1. Compactly supported data. In Section 4 we will discuss some mathematical properties of (3) and (4) when initial data have compact support. Here we display some computations on the domain $0<x<4$ with the compactly supported and infinitely differentiable initial data

$$
\phi(0, x)= \begin{cases}0 & 0<x \leq 1 \\ 2 e^{\frac{1}{(1-x)(3-x)}} & 1<x \leq 3 \\ 0 & 3<x<4\end{cases}
$$


Boundary operators satisfying the summation by parts property (see [15]) was added to the the sixth order first derivative approximation (6). Summation by parts boundary modification was also made for the second derivative operator (7); see [14. The boundary modifications reduce the order of accuracy to third at the boundary. For the elliptic equation (4), fifth order accuracy was observed in the computations. This is because of the smoothing property of the elliptic PDE improves the order by two.

Zero velocity conditions, $u(0, t)=u(4, t)=0$, were imposed at the boundaries of (4). No boundary condition was needed for (3), because the boundary is characteristic. In the computations below, $\eta=0.01$. The initial data together with the computed velocity are displayed in Figure 8.
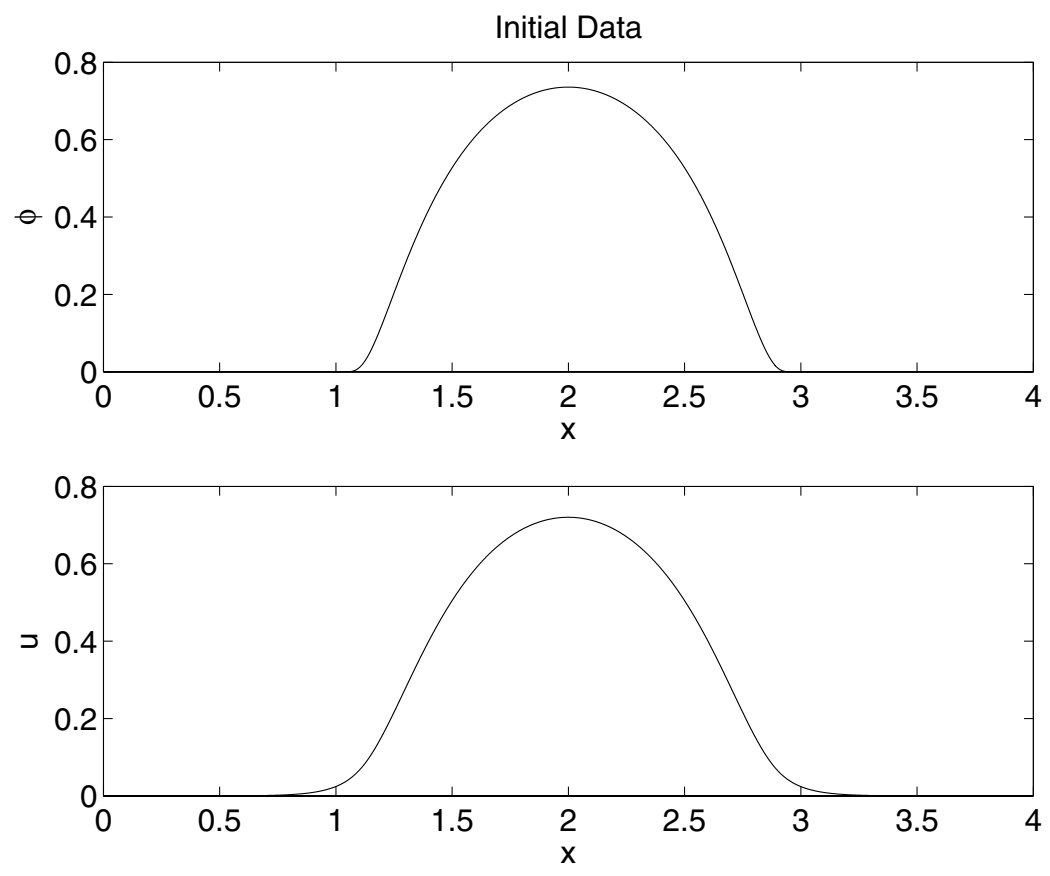

Figure 8. Compactly supported initial data, $\phi$ (upper) and $u$ (lower).

As time evolves, all mass travel with the positive velocity to the right and piles up at the right boundary of the support. There is no break up of the peak. The peak becomes higher and narrower with time, and the numerical solution eventually breaks down due to insufficient resolution. The solution at time 1.2 is displayed in Figure 9. The grid convergence can be assessed from Figure 10, where solutions with four different resolutions are compared. Note that the maximum in $\phi$ increases when refining from 4000 to 8000 points, and from 8000 to 16000 points, but is almost constant when refining from 16000 to 32000 points. As in the periodic case, it seems as if a smooth solution is captured if the resolution is fine enough. In the next section we will prove that this is true. 


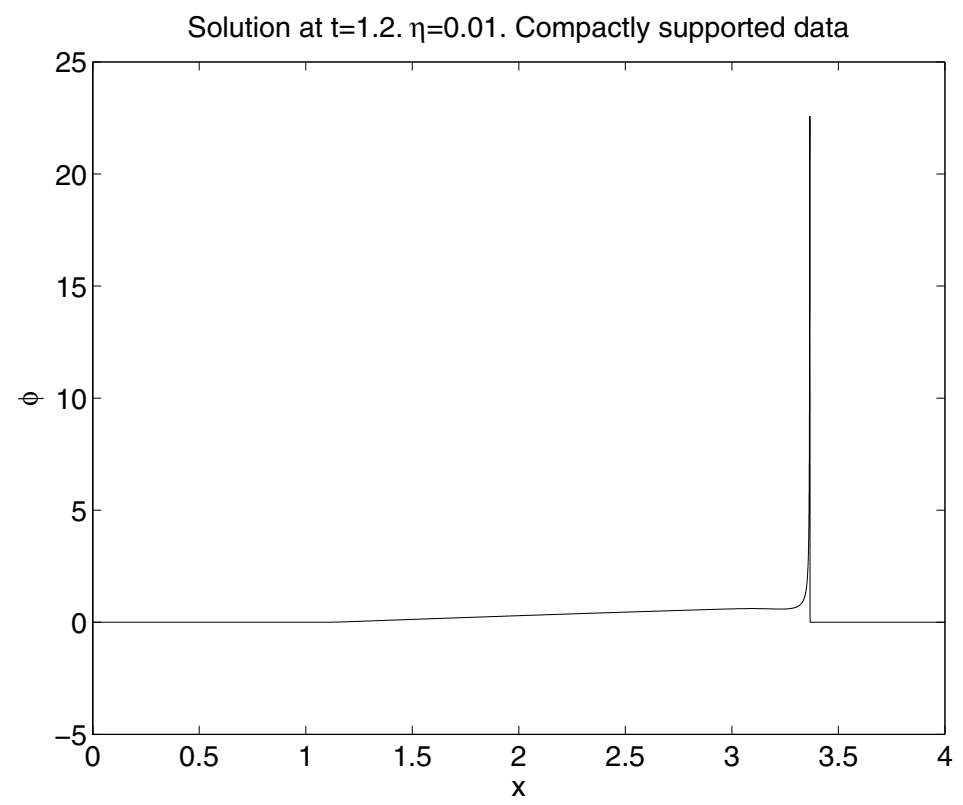

FIgURE 9. Solution at time 1.2.

Solution at $\mathrm{t}=1.2$ for $\eta=0.01$. Compactly supported initial data

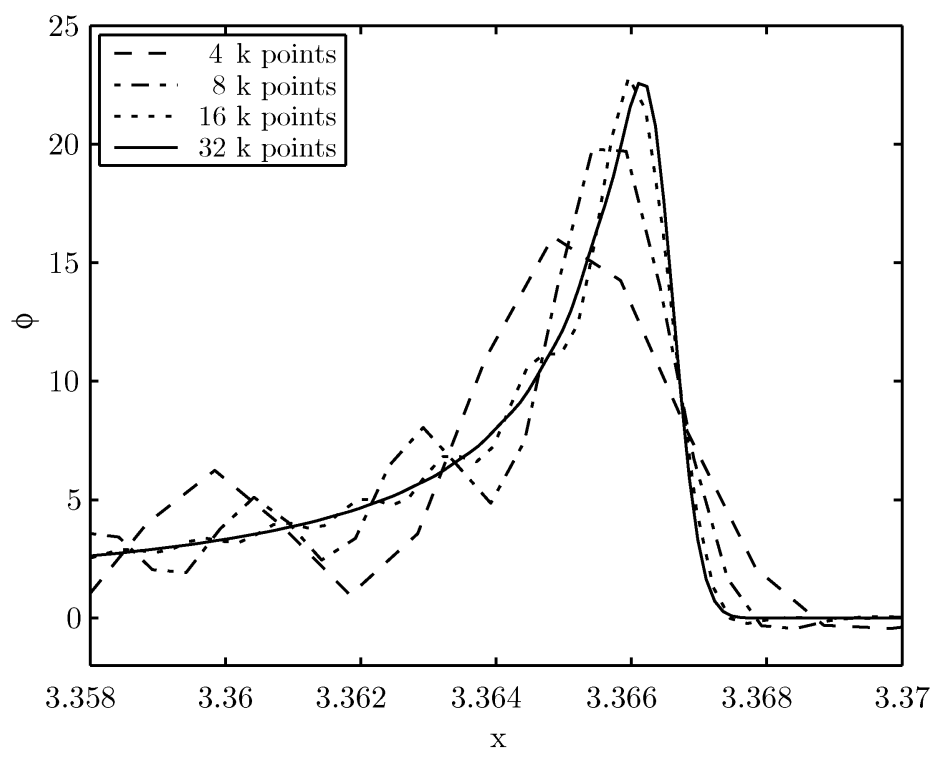

Figure 10. Grid convergence study at time 1.2. Close up of $\phi$ near $x=3.3$. 


\section{Existence of A SMOOTh SOLUtion}

The numerical experiments presented in Section 3 (see Figure 5 ) indicate that the solution of the model problem, (3)-(4), is smooth and that the spike-like behavior is not a singularity. In this section we show mathematically that the solution is smooth for any bounded time.

Consider again the model problem

$$
\begin{gathered}
\phi_{t}+(u \phi)_{x}=0, \quad-\infty<x<\infty, \quad t \geq 0, \\
-\eta u_{x x}+u=\phi, \\
\phi(0, x)=f(x),
\end{gathered}
$$

where $f(x)>0$ and is a smooth and compactly supported function and $\eta>0$ is a constant.

Formally, we can express the equation for $\phi$ as an ODE along a characteristic, $x(t)$ as

$$
\frac{d \phi(x(t), t)}{d t}=-u_{x}(x(t) ; t) \phi(x(t), t)
$$

where

$$
\frac{d \phi}{d t}=\frac{\partial \phi}{\partial t}+\frac{d x}{d t} \frac{\partial \phi}{\partial x} \quad \text { and } \quad \frac{d x}{d t}=u(x(t) ; t) .
$$

We have formulated Gronwall's lemma as follows.

Lemma 4.1. If

$$
\frac{d \phi(x(t), t)}{d t}=b(x(t), t) \phi+g(x(t), t)
$$

and $|b| \leq C_{b}$, then

$$
|\phi(\cdot, t)|_{\infty} \leq e^{C_{b} t}|\phi(\cdot, 0)|_{\infty}+e^{C_{b} t} \int_{0}^{t}|g(\cdot, s)|_{\infty} d s
$$

Theorem 4.2. For any bounded time, $0 \leq t \leq T$, the model problem given by equations (8) and (9) with $f$ smooth and compactly supported, has smooth solutions $\phi(x, t)$ and $u(x ; t)$ with any number of bounded derivatives. The bound of $\phi(x, t)$ is given by

$$
|\phi(\cdot, t)|_{\infty} \leq e^{\frac{K t}{\eta}}|f(\cdot)|_{\infty}
$$

while $u(x ; t)$ satisfies 


$$
|u(x ; t)| \leq \frac{K}{\sqrt{\eta}} .
$$

Here $K=\int_{-\infty}^{\infty} f(y) d y$.

Comment: From numerical experiments it seems like $|\phi| \rightarrow \infty$ as $\eta \rightarrow 0$ but with a slower rate than $e^{K / \eta}$; see Section 3 .

Proof. To show that a smooth solution exists, we use the following fixed point iteration, 12

$$
\begin{gathered}
\phi_{t}^{n+1}+u^{n} \phi_{x}^{n+1}=-u_{x}^{n} \phi^{n+1} \quad 0 \leq t \leq T, \quad n=0,1,2, \ldots, \\
\phi^{n+1}(x, 0)=f(x), \\
-\eta u_{x x}^{n}+u^{n}=\phi^{n},
\end{gathered}
$$

where $\phi^{0}(x, t) \equiv f(x)$.

Since $u^{n}(x ; t)$ is the solution to the elliptic equation, (18), it can be written as

$$
u^{n}(x ; t)=\left(G * \phi^{n}\right)(x, t) \quad \text { with } \quad G(x)=\frac{1}{\sqrt{\eta}} e^{-\frac{|x|}{\sqrt{\eta}}} .
$$

Thus

$$
\begin{aligned}
\left|u^{n}(x ; t)\right| & =\left|\int_{-\infty}^{\infty} G(x-y) \phi^{n}(y, t) d y\right| \\
& =\int_{-\infty}^{\infty} \frac{1}{\sqrt{\eta}} e^{-\frac{|x-y|}{\sqrt{\eta}}} \phi^{n}(y, t) d y \leq \frac{1}{\sqrt{\eta}} \int_{-\infty}^{\infty} \phi^{n}(y, t) d y=\frac{K}{\sqrt{\eta}},
\end{aligned}
$$

where we have used the fact that $\int_{-\infty}^{\infty} \phi^{n}(y, t) d y=K$ and that $\phi^{n} \geq 0$ for all times.

Similarly, we obtain for $\left|u_{x}^{n}(x ; t)\right|$,

$$
\begin{aligned}
\left|u_{x}^{n}(x ; t)\right| & =\left|\int_{-\infty}^{\infty} G^{\prime}(x-y) \phi^{n}(y, t) d y\right| \\
& =\int_{-\infty}^{\infty} \frac{1}{\eta} e^{-\frac{|x-y|}{\sqrt{\eta}}} \phi^{n}(y, t) d y \leq \frac{1}{\eta} \int_{-\infty}^{\infty} \phi^{n}(y, t) d y=\frac{K}{\eta} .
\end{aligned}
$$

If $\phi^{n}$ exists and is smooth, then $\left|u^{n}(x ; t)\right|$ is bounded according to (20) and $\left|u_{x}^{n}(x ; t)\right|$ is bounded according to (21). Hence the characteristic, $\frac{d x}{d t}=u(x(t) ; t)$, exists in the equation for $\phi^{n+1}$, (16), and we can use the estimate in Lemma 4.1 to obtain a bound for $\phi^{n+1}$ independent of $n$,

$$
\left|\phi^{n+1}(\cdot, t)\right|_{\infty} \leq e^{\frac{K t}{\eta}}|f|_{\infty} \quad \text { for } \quad 0 \leq t \leq T .
$$

Thus, $\phi^{n+1}(x, t)$ exists and is bounded for $0 \leq t \leq T$. Note that the bounds for $u^{n}$ and $\phi^{n}$ are independent of $n$.

The bound on $u^{n}$ also gives finite speed of propagation, so for bounded times, $\phi^{n+1}(x, t)$ has compact support for all $n$, and $\int_{-\infty}^{\infty} \phi^{n+1}(y, t) d y=K$.

We proceed in the same manner as above to obtain estimates for the derivatives of $\phi^{n+1}$. Denote the $j$-th derivative of $\phi^{n+1}$ by

$$
\phi_{j}^{n+1}=\frac{\partial^{j} \phi^{n+1}}{\partial x^{j}}, \quad j=1,2, \ldots, M .
$$


If we differentiate equation (16), we obtain

$$
\left(\phi_{j}^{n+1}\right)_{t}+u^{n}\left(\phi_{j}^{n+1}\right)_{x}=-(j+1) u_{x}^{n} \phi_{j}^{n+1}+g_{j}^{n} .
$$

Note that we cannot let $M \rightarrow \infty$.

The function $g_{j}^{n}$ contains only lower derivatives of $\phi^{n+1}$ and $u^{n}$. Any derivative, $u_{x x}^{n}$, or higher can be replaced by lower derivatives of $u^{n}$ by use of (9). This means that the bounds on $u^{n}$ and $u_{x}^{n}$ are sufficient. Note also that $\phi_{j}^{n+1}$ has compact support since it is the derivative of a compactly supported function.

From Lemma 4.1 we obtain by induction the estimate for the $j$-th derivative of $\phi^{n+1}$,

$$
\left|\phi_{j}^{n+1}(\cdot, t)\right|_{\infty} \leq e^{\frac{K t}{\eta}}\left|\phi_{j}^{n+1}(\cdot, 0)\right|_{\infty}+e^{\frac{K t}{\eta}} \int_{0}^{t}\left|g_{j}^{n}(\cdot, s)\right|_{\infty} d s \leq C_{j}(t)
$$

for $0 \leq t \leq T$ with $C_{j}(t)$ a constant independent of $n$.

Because of the bounds of $\phi^{n+1}$ and its derivatives, we can extract convergent subsequences $\left\{\phi^{n_{k}}\right\}$ such that for some smooth limit function $\phi$, it holds that $\lim _{n_{k} \rightarrow \infty}\left|\phi^{n_{k}}-\phi\right|_{\infty} \rightarrow 0$ and $\lim _{n_{k} \rightarrow \infty}\left|\phi_{j}^{n_{k}}-\phi_{j}\right|_{\infty} \rightarrow 0$. Here and from now on $|\cdot|_{\infty}$ denotes the maximum norm over space and time if nothing else is declared.

The next step is to show that the limit function, $\phi$, solves equation (8). This can not be done directly since we have terms $u_{x}^{n_{k}} \phi^{n_{k}+1}$ where $\phi^{n_{k}+1}$ is not necessarily in the subsequence. So, we need to show that $\lim _{n_{k} \rightarrow \infty}\left|u_{x}^{n_{k}} \phi^{n_{k}+1}-u_{x} \phi\right|_{\infty} \rightarrow 0$.

If $\lim _{n_{k} \rightarrow \infty}\left(\phi^{n_{k}+1}-\phi^{n_{k}}\right) \rightarrow 0$, then $\lim _{n_{k} \rightarrow \infty} u^{n_{k}-1} \phi_{x}^{n_{k}} \rightarrow u \phi_{x}$ since

$$
u_{x}^{n_{k}} \phi^{n_{k}+1}=u_{x}^{n_{k}} \underbrace{\left(\phi^{n_{k}+1}-\phi^{n_{k}}\right)}_{\rightarrow 0}+\underbrace{u_{x}^{n_{k}} \phi^{n_{k}}}_{\rightarrow u_{x} \phi}
$$

To show that $\lim _{n_{k} \rightarrow \infty}\left(\phi^{n_{k}+1}-\phi^{n_{k}}\right) \rightarrow 0$ let $w^{n+1}=\phi^{n+1}-\phi^{n}$. By equation (16) we obtain an equation for $w^{n+1}$ as

$$
\begin{aligned}
w_{t}^{n+1}+u^{n} w_{x}^{n+1}= & -u_{x}^{n} w^{n+1}-\phi_{x}^{n}\left(u^{n}-u^{n-1}\right) \\
& -\phi^{n}\left(u^{n}-u^{n-1}\right)_{x}, \\
w^{n+1}(x, 0)= & 0 .
\end{aligned}
$$

The last two terms in equation (27) can be bounded as

$$
-\phi_{x}^{n}\left(u^{n}-u^{n-1}\right)-\phi^{n}\left(u^{n}-u^{n-1}\right)_{x} \leq C\left|w^{n}\right|_{\infty} .
$$

Here $C$ depends on $K, T, \eta$ and $\left|f_{\infty}\right|$ but is independent of $n$ and we have used that $u^{n}-u^{n-1}=G *\left(\phi^{n}-\phi^{n-1}\right)=G * w^{n}$ and that $\left|u_{x}^{n}-u_{x}^{n-1}\right|_{\infty}+\left|u^{n}-u^{n-1}\right|_{\infty} \leq$ $C\left|w^{n}\right|_{\infty}$

Using Lemma 4.1 yields, for equation (27),

$$
\left|w^{n+1}(\cdot, t)\right|_{\infty} \leq C e^{\frac{K T}{\eta}} \int_{0}^{T}\left|w^{n}(\cdot, s)\right|_{\infty} d s
$$


since $w^{n+1}$ is zero at $t=0$. From equation (29) it follows that (see [12, Lemma 3.3.4)

$$
\left|w^{n}\right|_{\infty} \leq C_{1} \frac{(\tilde{C} T)^{n}}{n !}
$$

where $\tilde{C}=C e^{\frac{K T}{\eta}}$ and $C_{1}$ depends only on $\eta,|f|_{\infty}$, and on $K$. Hence $\left|w^{n}\right|_{\infty}=$ $\left|\phi^{n+1}-\phi^{n}\right|_{\infty} \rightarrow 0$ when $n \rightarrow \infty$.

We must also show that

$$
\lim _{n \rightarrow \infty}\left|\phi_{x}^{n+1}-\phi_{x}^{n}\right|_{\infty} \rightarrow 0 .
$$

To do this, differentiate equation (27) by

$$
\begin{aligned}
\left(w_{x}^{n+1}\right)_{t}+u^{n}\left(w_{x}^{n+1}\right)_{x}= & -2 u_{x}^{n} w_{x}^{n+1}-2 \phi_{x}^{n}\left(u^{n}-u^{n-1}\right)_{x} \\
& -\phi^{n}\left(u^{n}-u^{n-1}\right)_{x x}-\phi_{x x}^{n}\left(u^{n}-u^{n-1}\right), \\
w_{x}^{n+1}(0, x)= & 0,
\end{aligned}
$$

and similarly to the above we obtain $\left|w_{x}^{n}\right|_{\infty}=\left|\phi_{x}^{n+1}-\phi_{x}^{n}\right|_{\infty} \rightarrow 0$ when $n \rightarrow \infty$. So, the limit function, $\phi$ satisfies the model equation (8). This concludes the proof of Theorem 4.2 .

\section{ACKNOWLEDGMENTS}

The authors would like to thank the Swedish Foundation of Strategic Research (SFF) Multiphase Flow Program and the Parallel Scientific Institute (PSCI) for their financial support.

\section{REFERENCES}

[1] F.M. Auzerais, R.J. Jackson, and W.B. Russel, The resolution of shocks and the effect of compressible sediments in transient settling, J. of Fluid Mechanics 195 (1988), 437-462.

[2] R. Bürger, S. Evje, K. Hvistendahl Karlsen, and K.-A. Lie, Numerical methods for the simulation of the settling of flocculated suspensions, Chemical Engineering Journal 80 (2000), 91-104.

[3] M.C. Bustos, F. Concha, R. Bürger, and E.M. Tory, Sedimentation and thickening, phenomenological foundation and mathematical theory, Kluwer Academic Publisher, 1999. MR.1747460 (2002b:76101)

[4] M. Dorobantu, Numerical integration of $1 d$ consolidation models, Trita-na-9503, Department of Numerical Analysis and Computing Science, Royal Institute of Technology, 10044 Stockholm, SWEDEN, 1995.

[5] D.A. Drew and S.L. Passman, Theory of multicomponent fluids, Applied Mathematical Science, vol 135, Springer, 1999. MR.1654261 (99k:76143)

[6] Reynir Levi Gudmundsson, On the well-posedness of the two-fluid model for dispersed twophase flow in 2D, Report TRITA-NA-0223, Dept. of Num. Anal. and Comp. Sci., Royal Institute of Technology, Stockholm, Sweden, 2002.

[7] Reynir Levi Gudmundsson and Björn Sjögreen, Numerical experiments with two-fluid equations for particle-gas flow ii: Granular temperature effects, Report TRITA-NA-0442, Dept. of Num. Anal. and Comp. Sci., Royal Institute of Technology, Stockholm, Sweden, 2004.

[8] Reynir Levi Gudmundsson and Jacob Yström, Numerical experiments with two-fluid equations for particle-gas flow, Part of report TRITA-NA-0222, Dept. of Num. Anal. and Comp. Sci., Royal Institute of Technology, Stockholm, Sweden, 2002.

[9] Katarina Gustavsson and Björn Sjögreen, Numerical study of a viscous consolidation model, Hyperbolic Problems: Theory, Numerics, Applications (Thomas Y. Hou and Eitan Tadmor, eds.), Springer, 2002, pp. 569-578. MR2053206

[10] T. Hillen, C. Rohde, and F. Lutscher, Existence of weak solutions for a hyperbolic model of chemosensitive movement, Journal of Mathematical Analysis and Applications 260 (2001), 173-199. MR1843975 (2003c:35153) 
[11] Heinz-Otto Kreiss and Jacob Yström, Parabolic problems that are ill-posed in the zero dissipation limit, Mathematical and Computer Modelling 35 (2002), 1271-1295. MR.1910453 (2003e:35310)

[12] Heinz-Otto Kreiss and Jens Lorenz, Initial boundary values problems and the Navier-Stokes equations, Academic Press, 1989. MR0998379 (91a:35138)

[13] S. N. Kruzkov, First order quasilinear equations in several independent variables, Math. USSR. Sb. 10 (1970), 217-243.

[14] K. Mattson, Summation-by-parts operators for high order finite difference methods, Ph.D. thesis, Uppsala University, Information Technology, Department of Scientific Computing, 2003.

[15] B. Strand, Summation by parts for finite difference approximations for $d / d x$, J. Comput. Phys. 110 (1994), 47-67. MR 1259900 (94k:65126)

[16] M. Ungarish, Hydrodynamics of suspensions, Springer-Verlag, 1993.

[17] B.G.M. van Wachem, J.C. Shouten, C.M. van den Bleck, and J.L. Sinclair, Comparative analysis of CFD models of dense gas-solid systems, American Institute of Chemical Engineering Journal 47 (2001), 1035-1051.

[18] Jacob Yström, On two-fluid equations for dispersed incompressible two-phase flow, Comput. Visual. Sci. 4 (2001), 125-135. MR1946992(2003m:76175)

Royal Institute of Technology, 10044 Stockholm, Sweden

Current address: Center for Applied Scientific Computing, Lawrence Livermore National Laboratory, P.O. Box 808, L-550, Livermore, California 94551

E-mail address: sjogreen2@llnl.gov

Royal Institute of Technology, 10044 Stockholm, Sweden

E-mail address: katarina@nada.kth.se

Royal Institute of Technology, 10044 Stockholm, Sweden

E-mail address: rlg@nada.kth.se 